\title{
Miniaturized Nd-YAG laser as photoelastic transducer for small forces sensing
}

\author{
Naceur-Eddine Khelifa ${ }^{1, a}$ \\ ${ }^{1}$ LABORATOIRE COMMUN DE METROLOGIE, LNE-CNAM, 61 rue du Landy, case I36- 93210, La Plaine Saint-Denis, \\ France
}

\begin{abstract}
Measurement and traceability of small forces $(<0.5 \mathrm{~N})$ to the International System of Units (SI) remain a challenge for research and industry. For this purpose, we have developed a force sensor based on the photoelastic effect in a solid-state laser. At lower stress levels the induced shift in frequency between the two orthogonal polarizations, associated to a longitudinal laser mode, is proportional to the intensity of the force acting on the laser medium. From theoretical predictions, we show that high sensitivities can be obtained with miniaturized monolithic Nd-YAG laser. A short description of the used photoelastic force sensors is reported with a focus on his static response with an inventory of influencing parameters. The sensitive element of the force sensor is a plan-plan cylindrical monolithic Nd-YAG laser ( $2 \mathrm{~mm}$ in length and $3 \mathrm{~mm}$ in diameter). The overall uncertainty, estimated from beat frequencies measurements, comes mainly from reproducibility. Accordingly, with this kind of sensor it is possible to achieve high sensitivity required for measuring of small forces at the level of micronewton, but it still, at least for actual size sensor, more suitable for measurement in the range from $100 \mu \mathrm{N}$ to more than $1 \mathrm{~N}$.
\end{abstract}

Keywords. induced birefringence, photo-elastic effect, beat frequency, solid-state laser, force measurement, sensor sensitivity.

\section{Introduction}

La mise en œuvre de faibles forces intervient dans de nombreuses applications telles la microscopie à force atomique (AFM), les essais mécaniques pour la caractérisation des matériaux (micro et nanostructures), et dans le domaine de la biotechnologie. Toutes ces applications souffrent du fait que, les forces impliquées ne sont pas bien connues et surtout, ne sont pas traçable au "Système international d'unités" (SI).

$\mathrm{Au}$ niveau international, aucune référence, en dessous de $0,5 \mathrm{~N}$, n'est disponible. Pour remédier à cette situation, différents types d'instrumentations ont fait l'objet d'études et de comparaisons entre laboratoires [1-4]. Pour l'instant, aucun dispositif n'a été retenu pour constituer une voie vers une référence pour rendre traçable les forces en dessous de quelques dixièmes de newtons. Il faut noter que l'instrumentation destinée aux mesures de faibles forces continue de croître, notamment dans le domaine des sciences des matériaux et de la robotique, comme par exemple le développement d'instrumentations pouvant contrôler et ajuster finement le niveau de la force en fonction de l'objet manipulé [5].

Pour produire des faibles forces traçable au SI, le moyen direct est d'utiliser des masses étalons dans le champ de pesanteur (force désignée par le terme 'poids mort'). Il est en principe possible d'utiliser cette approche en produisant les étalons de masses adaptés à la plage de force recherchée. Cependant, la mise en pratique de cette méthode pose quelques difficultés techniques. En effet, lorsque le capteur de force est suffisamment petit (dimensions comparables à celles d'un poids mort comme dans le cas d'un cantilever d'AFM), il est difficile de charger de façon reproductible et avec précision le capteur de force.

La méthode, fondée sur l'effet photoélastique dans un laser, consiste à convertir une force mécanique en une fréquence. Même si elle a déjà fait l'objet d'un certains nombres de publications [6- 9] des développements avec des capteurs miniatures sont encore nécessaires. Malgré quelques difficultés techniques pour générer et transmettre une force dans une direction donnée, ce type de capteur de force reste très intéressant pour deux raisons: sa réponse linéaire (dans le domaine d'élasticité du matériau) et son temps de réponse relativement court.

Dans cet article, nous commencerons par faire un bref rappel sur la biréfringence induite par une contrainte mécanique dans un laser solide et son effet sur les fréquences des deux composantes de polarisations orthogonales associées au mode laser. Ensuite, nous décriront les dispositifs expérimentaux utilisés et en particulier le système à rails parallèles miniatures. Les

\footnotetext{
a Email de correspondance de l'auteur : naceur.khelifa@cnam.fr
} 
résultats de mesures obtenus avec le capteur photoélastique seront reportés et analysés dans une dernière partie.

\section{Biréfringence et effet photoélastique}

La biréfringence induite dans le milieu amplificateur d'un laser (cas du Nd-YAG) lorsque ce dernier est soumis à une contrainte mécanique se manifeste par des indices de réfraction différents selon que la polarisation de l'onde qui traverse le cristal est dans la direction de la force ou dans la direction orthogonale. Cette biréfringence est d'autant plus importante si la force appliquée $\left(F_{\text {ext }}\right)$ est quasiparallèle à un axe de contrainte principale (photoélasticité à deux dimensions). Dans ce cas, les directions principales de l'ellipsoïde des indices coïncident avec celles des contraintes.

L'expression de la biréfringence induite dans le Nd-YAG, représentée par la relation de contrainte optique, est alors donnée par la loi classique de Wertheim [10], soit :

$$
\Delta n=n_{\perp}-n_{/ /}=C_{P E}^{\left(\lambda_{q}\right)}\left(\sigma_{\perp}-\sigma_{/ /}\right)
$$

$\mathrm{Ou}, \sigma_{/ /}$et $\sigma_{\perp}$ sont les contraintes principales induites au centre du laser selon des directions orthogonale et parallèle à celle de la force extérieure.

$C_{P E}^{\left(\lambda_{q}\right)}$, est la constante photoélastique du YAG qui dépend faiblement de la longueur d'onde $\lambda_{q}$ associée au mode laser longitudinal d'ordre q.

Dans le cas ou la force extérieure est dans un plan diamétral du barreau, contenant les deux directions de contraintes principales, la conversion de cette dernière en contraintes internes s'écrit [11]:

$$
\sigma_{\perp}-\sigma_{/ /}=G \frac{F_{e x t}}{\ell \times d}
$$

$\ell$ et $d$, sont respectivement la longueur et le diamètre du laser solide à Nd-YAG.

$G$ est un facteur de forme, dont la valeur dépend des conditions géométriques du barreau Nd-YAG par rapport au faisceau laser de pompage optique à $808 \mathrm{~nm}$.

L'effet de la biréfringence sur le mode laser oscillant va correspondre à un déphasage entre les polarisations orthogonales du mode laser. Ce déphasage, engendré par l'effet photoélastique, est mesurable par l'écart $\Delta v=\left(v_{\perp}-v_{/ /}\right)$entre les fréquences des deux modes de polarisations.

Cet écart en fréquence s'écrit : $\Delta v=c(\Delta n) / n \lambda_{q}$

Ou, $n$ est l'indice de réfraction du Nd-YAG sans

contrainte et ${ }^{c}$ la vitesse de la lumière dans le vide.

En utilisant les relations (1) et (2), cet écart en fréquence s'exprime en fonction de la force extérieure par :

$$
\Delta v=G \frac{v_{q} C_{P E}^{\left(\lambda_{q}\right)}}{n \ell d} F_{e x t}
$$

$v_{q}=c / \lambda_{q}$ est la fréquence d'émission du laser. La sensibilité du capteur est alors définie par $\Delta v / F_{e x t}$.

Dans le cas idéal, le faisceau laser de pompe doit être d'une part, de faibles dimensions par rapport au diamètre du laser Nd-YAG et d'autre part, centré sur l'axe de révolution de ce dernier. Comme il sera montré dans la section 4 , cette double condition devient critique et par conséquent difficile à réaliser lorsqu'on utilise des lasers monolithiques miniatures.

Dans la configuration géométrique idéale, le facteur $G$

vaut $8 / \pi$. En considérant les valeurs [9] des différentes constantes, la relation (3) de la sensibilité théorique s'exprime simplement par :

$$
S_{1}^{T h}=\frac{492}{\ell \times d}
$$

Pour des raisons de commodité, les grandeurs $\ell$ et $d$ dans l'expression (4) sont exprimées en $\mathrm{mm}$ pour que la sensibilité $S_{1}^{T h}$ soit en $\mathrm{MHz} \times \mathrm{N}^{-1}$.

\section{Montages expérimentaux}

\section{1- Principe de l'expérience}

Le montage expérimental, du capteur photoélastique, utilise un laser monolithique $\mathrm{Nd}-\mathrm{YAG}(2 \mathrm{~mm} \times 3 \mathrm{~mm})$ à résonateur plan-plan. Ce dispositif a déjà fait l'objet d'une description assez détaillée [12]. La figure 1 présente le schéma de principe de l'expérience. La force est appliquée verticalement sur le barreau laser, soit à l'aide de poids morts (masse étalon) soit contact avec la surface sphérique d'une céramique fixée à l'extrémité d'un actionneur piézoélectrique à déplacement nanométrique.

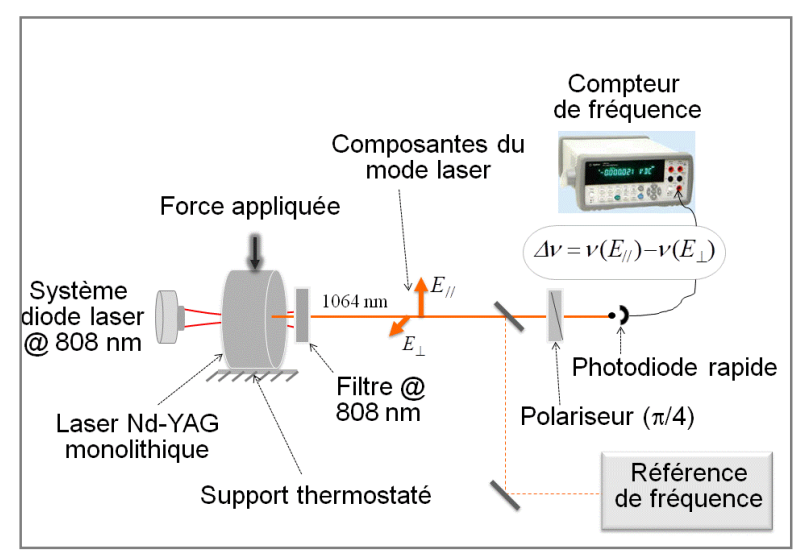

Figure 1. Schéma de principe du capteur photoélastique de force

\section{2- Transducteur sur rails parallèles miniatures}

Un actionneur piézoélectrique, en mode transducteur, convertit un signal électrique en un déplacement physique, contrôlable au niveau nanométrique. En général, il est utilisé pour effectuer un déplacement mais on peut également restreindre son déplacement pour produire une force. Sous contrainte élastique, la force maximum (force bloquée) que génère l'actionneur dépend de son 
déplacement maximum mais également de sa raideur et de celle du matériau qui restreint le déplacement.

$$
F_{\max } \cong k_{a} \times \Delta X_{\max }\left(1-\frac{k_{a}}{k_{a}+k_{m}}\right)
$$

$k_{a}$ et $k_{m}$ sont respectivement les raideurs en $\left[\mathrm{N} \times \mathrm{m}^{-1}\right]$ de l'actionneur piézoélectrique et du cristal Nd-YAG fixé sur son support rigide plan; $\Delta X_{\max }$ est le déplacement maximum généré par l'actionneur sans contrainte extérieure.

Ainsi, selon la situation expérimentale et les ordres de grandeurs des raideurs la force générée par le transducteur PZT peut aller de $F_{\max }$ (plusieurs newtons) à quasiment 0 newtons.

Dans notre expérience, l'élément piézoélectrique (PZT) utilisé est un actionneur de grande résolution à jauge de contrainte fonctionnant en boucle fermée afin de réduire l'effet d'hystérésis (déplacement avec une résolution de l'ordre du $\mathrm{nm}$, bande passante de quelques $\mathrm{kHz}$ ).

Cet élément PZT est porté par un dispositif à rails miniature parallèles. Ce mécanisme de guidage permet de positionner l'embout, en céramique, du transducteur à quelques micromètres de la partie sensible du capteur photoélastique de force. La figure 2 montre une photographique du transducteur PZT et du système de guidage par rails miniatures.

Le positionnement final de l'extrémité sphérique de la PZT par rapport au laser est réalisé par application d'une tension continue qui commande le moniteur de l'actionneur piézoélectrique. Pour différentes valeurs de cette tension continue, nous avons analysé la reproductibilité de la réponse du capteur photoélastique et de la force de contact entre le barreau Nd-YAG et la partie sphérique du transducteur. Ce système a été développé pour générer également de faibles forces dynamiques mais ne sera pas présenté dans ce papier.

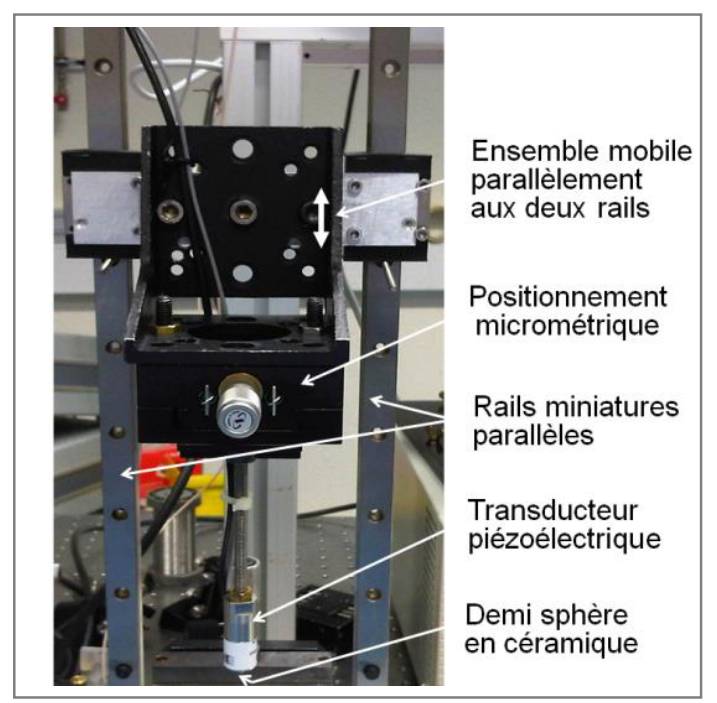

Figure 2. Système de guidage du transducteur piézoélectrique

\section{Résultats expérimentaux}

\section{1- Réponse du capteur aux faibles poids morts}

La charge du laser par une masse étalon $m_{e}$ va engendrer dans le champ local de pesanteur $\vec{g}_{\text {loc }}$, une force $\vec{F}_{g}=m_{e} \times \vec{g}_{l o c}$. La mesure de $g_{l o c}$ avec une grande exactitude permet de traduire une masse étalon en poids mort [9]. Ainsi, pour la conversion de la sensibilité de $\mathrm{MHz} \times \mathrm{g}^{-1}$ en $\mathrm{MHz} \times \mathrm{N}^{-1}$, la relation suivante est utilisée :

$$
1 \mathrm{MHz} \times \mathrm{N}^{-1} \cong 9,80878 \times 10^{-3} \mathrm{MHz} \times \mathrm{g}^{-1}
$$

Dans une première campagne de mesure de la sensibilité du capteur photoélastique [12], nous avons effectué une série de mesure de charge-décharge de l'élément sensible du capteur à l'aide de masses étalons allant de $0-20 \mathrm{~g}$. A partir de la courbe de réponse obtenue, nous avons déterminé la valeur de la sensibilité du capteur aux faibles charges (inférieures à $50 \mathrm{mN}$ ).

$$
S(0-5 g)=57,97(1,21) \mathrm{MHz} \times \mathrm{N}^{-1}
$$

Ici, nous avons utilisé la même procédure pour la mesure de la sensibilité du capteur photoélastique lorsqu'il est soumis à différents poids morts, mais en utilisant cette fois-ci des masses étalons comprises entre $0.1 \mathrm{~g}$ et $2 \mathrm{~g}$. La réponse du capteur aux faibles charges est représentée sur la figure 3. Ces résultats montrent, que pour des contraintes relativement faibles, l'écart en fréquence induit entre les deux polarisations orthogonales d'un mode longitudinal, est proportionnel à la contrainte appliquée.

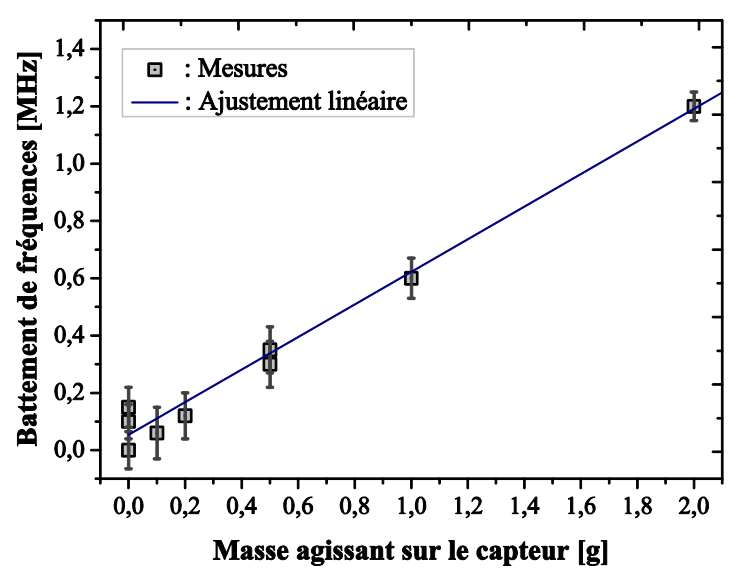

Figure 3. Réponse du capteur en fonction de sa charge.

La sensibilité expérimentale, dans la gamme de charge étudiée, est déterminée à partir de l'ajustement linéaire des résultats de mesures. La pente de la courbe représentée sur la figure 3 et son incertitude exprimée par un écart-type est

$$
\begin{aligned}
& : \quad 0,569 \quad(0,008){\mathrm{MHz} \times \mathrm{g}^{-1},}, \quad \text { soit encore: } \\
& S(0-2 g)=58,01(0,82) \mathrm{MHz} \times \mathrm{N}^{-1}
\end{aligned}
$$

Comme on peut le constaté, les valeurs obtenues pour la sensibilité présentent entre elles un écart relatif inférieur à $10^{-3}$.

Il faut savoir que depuis la première mesure de sensibilité, le capteur a été soumis à de nombreuses contraintes tout en 
restant dans le domaine d'élasticité du Nd-YAG. De ce fait, nous pouvons affirmer que le comportement du laser monolithique, utilisé comme capteur de force durant plus de six mois, semble ne pas présenter des signes apparents de vieillissement sur cette période.

\section{2- Sensibilité du capteur de force}

Les valeurs de sensibilité obtenues avec le capteur $(2 \mathrm{~mm}$ x $3 \mathrm{~mm}$ ), reportées sur la figure 4, ont permis d'affiner au mieux la valeur du paramètre de forme G. Il s'agissait de réduire les écarts importants observés entre les prévisions du modèle théorique utilisé et les valeurs d'expériences. Les écarts sont particulièrement significatifs dans le cas de capteurs de faibles dimensions. Les prévisions du modèle théorique, utilisé précédemment, sont indiquées sur la figure 4 par la courbe notée $S_{1}^{\text {th }}$.

La sensibilité théorique du capteur photoélastique donnée par l'expression (1) n'est pas en accord avec toutes les mesures. C'est la valeur du paramètre $\mathrm{G}$, que nous avons considérée, qui peut expliquer cette observation.

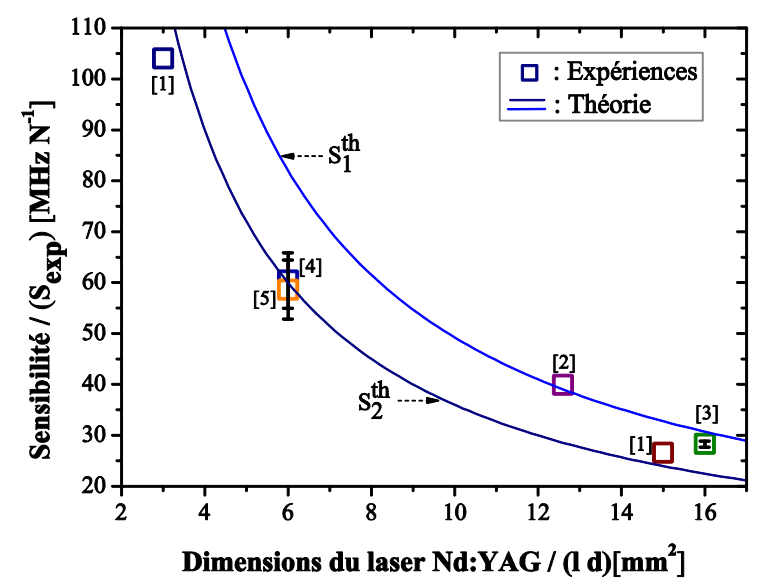

Figure 4. Sensibilités observées pour des capteurs laser $\mathrm{Nd}-\mathrm{YAG}$ de différentes dimensions.

En effet, $\mathrm{G}$ est un facteur qui dépend non seulement de la nature du contact entre le barreau laser et son support mais aussi de son orientation par rapport au faisceau laser utilisé pour réaliser l'inversion de population dans le laser $\mathrm{Nd}$ YAG.

Ainsi, comme nous l'avons fait remarquer dans la section 2, la valeur de $\mathrm{G}$ devient critique pour un capteur laser de faibles dimensions. L'approximation ( $G=8 / \pi$ ), qui a été faite, n'est plus acceptable dans la mesure les conditions d'alignement et de centrage du faisceau de pompe par rapport au barreau laser sont difficiles à réaliser et à maintenir au cours des mesures. En effet, si la force de contact exercée par le support sur le barreau laser n'est plus orthogonale et dans le plan diamétral, ou un léger décentrage du faisceau pompe par rapport à l'axe de révolution du barreau, alors cela va affecter G. En gardant le même modèle théorique et en ajustant la valeur de $\mathrm{G}$ de telle sorte à reproduire au mieux les mesures d'expériences, nous avons abouti au résultat représenté par la courbe $S_{2}^{T h}$ comme indiquée sur la figure 4. Ce résultat correspond à la sensibilité donnée par la relation (3) en considérant la valeur de $6 / \pi$ pour le facteur $G$.

Dans le cas ou l'émission laser contient plus d'un mode longitudinal, l'acquisition et le traitement du signal de battement n'est pas simple, notamment lorsque la force appliquée sur le capteur est variable (dynamique). La configuration idéale du laser $\mathrm{Nd}-\mathrm{YAG}$ est celle qui correspond à un fonctionnement monomode longitudinal. Dans ce cas le compteur de fréquence indique une seule valeur et donc facile à suivre et à exploiter. La largeur à mi-hauteur de la courbe de gain du Nd-YAG étant de l'ordre de $120 \mathrm{GHZ}$, la longueur du résonateur formé par les deux faces du barreau Nd-YAG doit vérifier la condition : $c /(2 n \ell)>120 \mathrm{GHz}$

$c$ étant la vitesse de la lumière dans le vide et $\mathrm{n}$ l'indice de réfraction du YAG. Par conséquent, pour un fonctionnement monomode, la longueur de la cavité laser doit être inférieure à $\ell<0,7 \mathrm{~mm}$ (configuration d'un laser Nd-YAG miniature).

Ainsi, pour un capteur photoélastique, réalisé avec un tel laser " miniature », nous obtiendrons par extrapolation à partir de la courbe théorique $\left(S_{2}^{\text {th }}\right)$, une sensibilité supérieure à $300 \mathrm{MHz} \times \mathrm{N}^{-1}$.

Sur la figure 5 sont reportées les réponses de trois capteurs aux très faibles charges (inférieures à $5 \mathrm{mN}$ ). Cette figure montre bien que la mesure des faibles forces est plus accessible en raison de la grande sensibilité pouvant être atteinte.

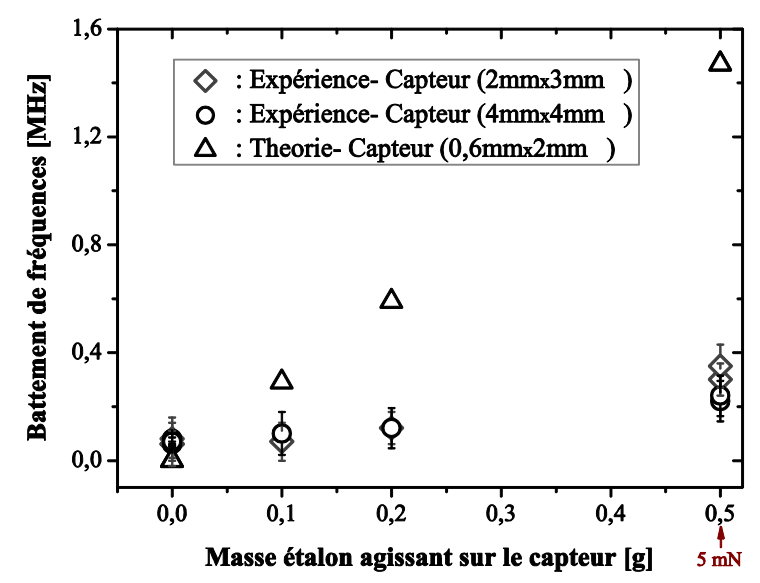

Figure 5. Extrapolation de la réponse aux faibles poids morts d'un capteur laser miniature

Un premier test du dispositif, utilisant deux rails profilés miniatures, a été fait pour vérifier la réponse de l'actionneur PZT à une tension continue fixe. Le positionnement de l'extrémité du transducteur par rapport au haut du barreau laser reste assez critique mais relativement simple à réaliser. Sur la figure 6 sont reportées les valeurs du battement de fréquences obtenues pour une série de charge-décharge du capteur photoélastique lorsque la tension de commande du moniteur de l'actionneur PZT est fixée successivement à $0 \mathrm{~V}$ puis à $5 \mathrm{~V}$. Ces valeurs correspondent à la réponse globale du capteur photoélastique et de l'actionneur 
piézoélectrique. Les fréquences moyennes du signal de battement de fréquences et l'incertitude de reproductibilité sont :

$$
\left\{\begin{array}{l}
\overline{\Delta v}_{0}(\mathrm{~V}=0 \text { volts })=0.063(0.045) \mathrm{MHz} \\
\overline{\Delta v_{5}}(\mathrm{~V}=5 \text { volts })=13,183(0.138) \mathrm{MHz}
\end{array}\right.
$$

Pour évaluer la force de contact exercée par l'extrémité du transducteur sur le capteur, on utilise la sensibilité expérimentale qui a été déterminée par raccordement à des étalons de masses (section 4.1), soit :

$$
S(0-2 g)=58,01(0,81) \mathrm{MHz} \times \mathrm{N}^{-1}
$$

La force générée, par la déformation de l'actionneur piézoélectrique sous l'action d'une tension électrique, est appliquée sur l'élément sensible du capteur photoélastique de force. La traduction de cette force en termes de fréquence est donnée par la figure 7. Cette dernière montre que la réponse du capteur de force est quasi-linéaire.

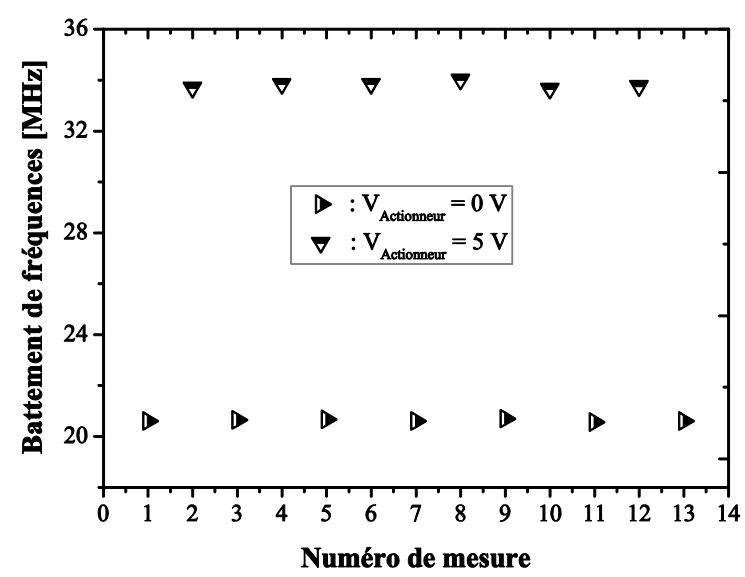

Figure 6. Réponse du capteur photoélastique à la force générée par l'actionneur piézoélectrique.

Le déplacement en $\mu \mathrm{m}$ en fonction de la tension de commande du transducteur $(0-10 \mathrm{~V})$ étant linéaire à mieux que $0.1 \%$, nous avons vérifié celle du capteur photoélastique. Sur la figure 7, sont reportées les valeurs moyennes du battement de fréquences observées lorsque l'actionneur PZT, commandé par une tension continue de 0 à $10 \mathrm{~V}$, agit sur le capteur.

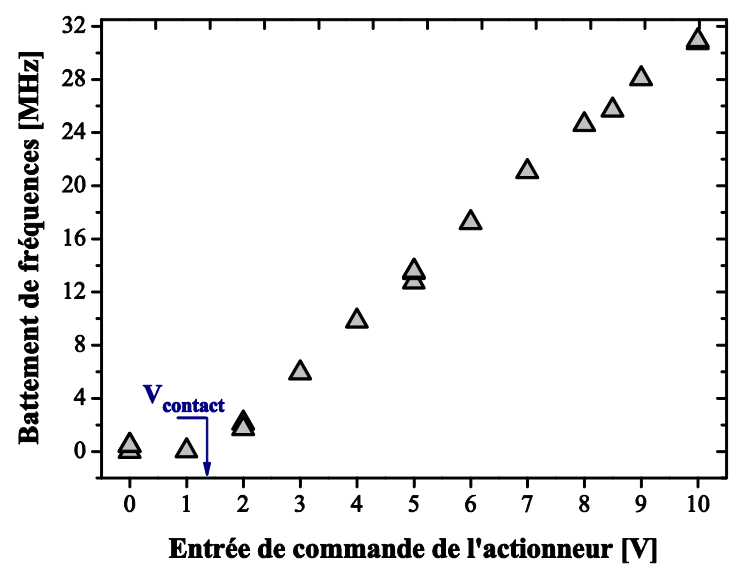

Figure 7. Réponse du capteur photoélastique aux contraintes engendrées par l'actionneur piézoélectrique.

Ainsi, la force maximale exercée par le transducteur sur le capteur est de l'ordre de : $F_{\max }=523 \mathrm{mNavec}$ une incertitude de répétabilité de l'ordre de $18 \mathrm{mN}$.

En perspective, nous avons démarré une étude de la réponse du capteur photoélastique à une force dynamique de forme et d'intensité connues générée par une tension de commande sinusoïdale $\mathrm{du}$ transducteur PZT. Les premières observations sont prometteuses mais en revanche, une méthode élaborée pour l'acquisition du signal de battement de fréquences reste à mettre en œuvre.

\section{Conclusion}

Les mesures de sensibilités du capteur photoélastique de force $(2 \mathrm{~mm} \times 3 \mathrm{~mm})$ et les valeurs reportées par d'autres équipes ont permis d'identifier les différents paramètres pouvant influencer ses caractéristiques. La miniaturisation du capteur de force est souhaitable car elle correspond à la configuration où d'une part, l'acquisition du signal de battement de fréquences est moins laborieuse et d'autre part, de gagner en sensibilité. Les premiers tests de ce capteur, lorsqu'il est soumis à une force générée par un transducteur piézoélectrique montrent qu'il reproduit parfaitement la déformation du transducteur sans aucun effet d'hystérésis. Avec ce dernier système, nous avons entrepris une caractérisation du capteur en mode d'excitation dynamique par une force périodique de quelques $\mathrm{Hz}$ à plus de $1 \mathrm{kHz}$.

\section{Références}

1. S. Niche, Journal of Metrology of India, 20, pp. 239248, (2005).

2. W. Dong; D. Rostoucher and M. Gauthier; Rev. Sci. Instrum. 81, 116101, (2010). http://dx.doi.org/10.1063/1.3488382

3. Ch. Schlegel; O. Slanina; G. Haucke and R. Kumme IMEKO 2010 TC3 Conferences Metrology in Modern Context, November (2010), Thailand.

4. M-S. Kim; J. R. Pratt; U. Brand and Ch. W. Jones, Metrologia 49, pp.70-81, (2012). doi:10.1088/0026-1394/49/1/011

5. Ge Yang; M.A. Greninger; B.J. Nelson; ICRA 02, IEEE International Conference, Vol. 2, pp.1943, (2002)

6. Holzapfel, S. Neuschaefer-Rube and M. Kobusch, Measurement 28, pp. 277-291, (2000). http://dx.doi.org/10.1016/S0263-2241(00)00042-7

7. W. Holzapfel; M. Suske, XVII IMEKO World Congress TC3, (2006).

8. J. Ding, L.Zhang, Z. Zhang and S. Zhang, Opt. \& Laser Technol. 42, pp. 341-346, (2010). http://dx.doi.org/10.1016/j.optlastec.2009.08.002

9. N. Khelifa, Optics and Photonics Journal 4, $\mathrm{n}^{\circ} 1$, pp. 14-20, (2014). http://dx.doi.org/10.4236/opj.2014.41003. 
10. M. G. Wertheim, Ann. Chim. Phys. 40, 156-221 (1854)

11. M. M. Frocht, Phtoelasticity, $8^{\text {th }}$ edition, John Wiley and sons, London (1941), pp.144- 349.

12. Naceur-Eddine Khelifa, Marc Himbert, Sensors \& Transducers Journal, 184, pp. 19-25 (2015).

http://www.sensorsportal.com/HTML/DIGEST/P 25 $\underline{81 . h t m}$ 